\title{
Multi parameter flow cytometric assessment of regenerative epidermis with special reference to the antiproliferative effect of occlusion
}

\author{
M.M. van Rossum *, J.M. Mommers, P.C.M. van de Kerkhof, G.J. de Jongh, \\ C.A.E.M. van Hooijdonk and P.E.J. van Erp \\ Department of Dermatology, University Hospital Nijmegen, the Netherlands
}

Received 5 June 1998

Revised 8 October 1998

Accepted 15 October 1998

\begin{abstract}
Multi parameter flow cytometrical assays permit simultaneous assessment of proliferation, differentiation, and inflammation parameters. In this study, the validation of TO-PRO-3 iodide (TP3) compared to propidium iodide (PI) and DE-K10 compared to RKSE60 were evaluated in tape stripping induced hyperproliferation. No occlusion, Duoderm (intermediate occlusion) and Blenderm (maximal occlusion) were used as a model to evaluate the effect of occlusion on epidermal regeneration. Proliferation in the keratin 10-negative compartment measured with TP3 proved to be a good approximation of proliferation measured with PI. Other epidermal subpopulations (keratin 10-dim and -bright cells) did not make a relevant contribution to hyperproliferation. DE-K10 is probable more sensitive than RKSE60 to distinguish populations that differ in degree of differentiation. Occlusion of tape stripped skin resulted in decreased proliferation and increased differentiation. This effect was most pronounced with maximal occlusion. This study showed that occlusion is a therapy, which realises normalisation of hyperproliferative skin disorders.
\end{abstract}

\section{Introduction}

Human epidermis is a continuously renewing epithelium and it is known to be composed of subpopulations that differ with respect to proliferative and morphological characteristics $[1,16,21]$. Some of these subpopulations respond rapidly after a proliferative stimulus, whereas others are slow responders. Flow cytometrical characterisation of epidermal subpopulations proved to be a reproducible and quantitative approach to assess the epidermal changes in hyperproliferative skin [3,5,20]. Previously, multi parameter flow cytometry was used to quantify the percentages of suprabasal keratinocytes (keratin 10-positive cells), germinative cells (keratin 10-negative cells), and non-keratinocytes (vimentin-positive cells). In these studies, TP3 was used to measure DNA content per cell $[12,13]$. Although this methodology proved to be adequate to estimate hyperproliferative changes, a reappraisal of the validity of this methodology

\footnotetext{
${ }^{*}$ Correspondence: M.M. van Rossum, Department of Dermatology, University Hospital Nijmegen, P.O. Box 9101, NL-6500 HB Nijmegen, the Netherlands. Tel.: +31 24 3617245; Fax: +31 243541184.
} 
with two modifications was carried out in regenerative skin following tape stripping with and without occlusion.

The aim of the present study was to assess characteristics of regenerative epidermis with special reference to the antiproliferative effect of occlusion. Therefore, the result of two different probes (TP3 and PI) to assess DNA distribution and two different antibodies (RKSE60 and DE-K10) to assess keratin 10 expression in hyperproliferative skin with and without occlusive treatment were compared. The in vivo model for epidermal hyperproliferation was the tape stripping method. This method has been used as a standardised approach since 1951, and is well accepted as a procedure to induce hyperproliferation in normal skin [17]. With respect to the development of the flow cytometric methodology, we addressed the following questions:

- To what extent is the hyperproliferative activity in epidermal subpopulations measured with TP3 a reflection of total proliferation as measured by PI?

- Which antibody against keratin 10 (RKSE60 or DE-K10) has the highest sensitivity to distinguish epidermal subpopulations, which differ in degree of differentiation?

- To what extent does the keratin 10-dim (weakly positive) subpopulation contribute to regenerative epidermal proliferation?

One of the therapeutical options for psoriasis is occlusive treatment. This therapy stimulates regeneration of the disturbed granular layer [11] and reduces the mitotic rate $[4,11,24]$, whereas immunologic abnormalities remain unchanged [14,24]. The results with regard to the effect of occlusion on proliferation in tape stripped human skin are not uniform. Whereas Van de Kerkhof et al. found no differences with respect to number of cells in $\mathrm{SG}_{2} \mathrm{M}$-phase between uncovered and occluded skin $48 \mathrm{~h}$ after tape stripping [22], others showed that occlusive dressings decreased the mitotic activity of human skin after tape stripping $[8,25]$. Methodological limitations might account for this difference. The question with respect to antiproliferative therapy was the following: to what extent does the recently developed flow cytometrical assessment permit the quantification of the antiproliferative effect of occlusion, with a plastic film and a hydrocolloid, in regenerative skin following tape stripping?

\section{Material and methods}

\subsection{Subjects}

In this study 8 healthy volunteers participated. This group, aged between 22 and 58 years, consisted of two females and 6 males without signs or previous history of chronic inflammatory skin disease.

\subsection{Sellotape stripping and biopsy procedures}

After informed consent and permission of the ethics committee were obtained, three areas on the back (about $1.5 \times 2 \mathrm{~cm}$ ) were marked with a waterproof pencil. Sellotape (Sellotape gb Ltd., Dunstable, England) was applied with gentle pressure to the marked area, and then removed [17]. This procedure was repeated until the skin glistened. One of the stripped areas was left uncovered, whereas the others were occluded with an adhesive polyethylene film (Blenderm; 3M, Zoeterwoude, the Netherlands) and a hydrocolloid dressing (Duoderm; ConvaTec, Woerden, the Netherlands). After $48 \mathrm{~h}$ local anaesthesia was induced with xylocaine/adrenaline 1:100000 (Astra Pharmaceutica BV, Zoetermeer, the Netherlands), and one punch biopsy $(3 \mathrm{~mm})$ was taken from each tape stripped area. 


\subsection{Cell isolation procedure}

The tissue was preserved in phosphate-buffered saline (PBS) until cell isolation, for maximal $8 \mathrm{~h}$, as described previously [12]. In brief, the biopsies were kept in PBS (with calcium and magnesium) containing $0.5 \mathrm{mg} / \mathrm{ml}$ protease type x (Sigma, St. Louis, Mo., USA) at $4^{\circ} \mathrm{C}$ during $16-20 \mathrm{~h}$. Subsequently, the dermis and epidermis were separated with two forceps, and the epidermis was incubated for $30 \mathrm{~min}$ at $37^{\circ} \mathrm{C}$ in PBS containing $0.025 \mathrm{mg} / \mathrm{ml}$ trypsin type III (Sigma) and $0.3 \mathrm{mg} / \mathrm{ml}$ dithioerythritol (Sigma). After $10 \%$ foetal calf serum was added, the epidermis was gently mixed on a vortex to detach the keratinocytes. After the stratum corneum was removed, the cells were pelleted and fixed in ice-cold ethanol $(70 \% \mathrm{v} / \mathrm{v})$ and stored at $-20^{\circ} \mathrm{C}$.

\subsection{Flow cytometric analysis}

A triple-labelling technique was used to obtain simultaneously information about proliferation, differentiation and inflammation in tape stripped skin with and without occlusion. Reference values in normal skin are $7 \%$ vimentin-positive cells, 40 to $50 \%$ keratin 10-negative cells, 50 to $60 \%$ keratin 10-positive cells (among which are very few keratin 10-dim cells), and 5 to $10 \%$ basal cells in $\mathrm{SG}_{2} \mathrm{M}$ phase. The staining procedure was described previously [13]. In brief, inflammation was assessed by using Vim3B4 (Novocastra Laboratories, Newcastle upon Tyne, UK), a monoclonal antibody directed against vimentin. Differentiation was assessed using the monoclonal antibody RKSE60 (a gift from Prof. F.C.S. Ramaekers, Department of Molecular Biology, University of Maastricht, the Netherlands) or DEK10 (ICN Biomedicals BV, Zoetermeer, the Netherlands) directed against keratin 10. The fluorochromes TP3 (Molecular Probes, Eugene, USA) and PI (Omnilabo International BV, Breda, the Netherlands) were used for measuring DNA content per cell. Samples were divided into three parts: one was measured with a combination of VIM3B4/RKSE60/TP3, the second with VIM3B4/DE-K10/TP3, and in the last DNA content was measured with PI. As secondary antibodies we used goat anti-mouse (GAM) IgG2a conjugated with fluorescein-isothiocyanate (FITC) (Southern Biotechnology Associates, Birmingham, USA) and goat anti-mouse IgG1 conjugated with phycoerythrin (PE) (Southern Biotechnology Associates) binding to Vim3B4 and RKSE60/DE-K10, respectively.

A sample containing about $2 \times 10^{5}$ cells was taken from each cell suspension, and washed with PBS. The suspension was centrifuged during $5 \mathrm{~min}$ at $3000 \mathrm{rpm}$. Subsequently, $150 \mu \mathrm{l}$ Vim3B4 (diluted 1:15 in PBS) and $150 \mu \mathrm{l} \mathrm{RKSE60} \mathrm{(diluted} 1: 5$ in PBS) or $150 \mu \mathrm{l} \mathrm{DE-K10} \mathrm{(diluted} 1: 100$ in PBS) were added, and the suspension was incubated in the dark for $30 \mathrm{~min}$ at room temperature. Subsequently, the cells were washed with PBS containing $1 \%$ heat-inactivated newborn calf serum (HINCS) and centrifuged. A solution of GAM-FITC (diluted 1:50), GAM-PE (diluted $1: 250$ ), normal goat serum and PBS/HINCS was added. After incubation on ice for $15 \mathrm{~min}$ the cells were washed with PBS/HINCS, centrifuged and resuspended in $300 \mu \mathrm{l}$ PBS containing TP3 $\left(2 \times 10^{-6} \mathrm{M}\right)$ and $50 \mu \mathrm{l} 1 \%$ RNase solution. DNA-content per cell was also measured with PI $(20 \mathrm{mg} / \mathrm{ml})$ for all samples.

An EPICS ${ }^{\circledR}$ Elite flow cytometer (Coulter, Luton, UK) was used to measure 5000 gated cells. An argon ion laser (15 mW, $488 \mathrm{~nm})$ excited FITC and PE, and a helium-neon laser (10 mW, $633 \mathrm{~nm})$ excited TP3. Bandpass filters of 515-535 nm (green, FITC), 555-595 nm (orange, PE), 665-685 nm (red, TP3), and a longpass filter of $630 \mathrm{~nm}$ (red, PI) were used to measure emitted light. The area/peak ratio in the DNA-histogram was used to exclude doublets of diploid cells and gate the real single tetraploid cells for further analysis [2]. The percentage of cells positive for vimentin was assessed by using gates. With respect to keratin 10 , cells were classified by gates as negative, dim (weakly positive) or bright 
Measurement of cells in S-phase with different DNA-probes

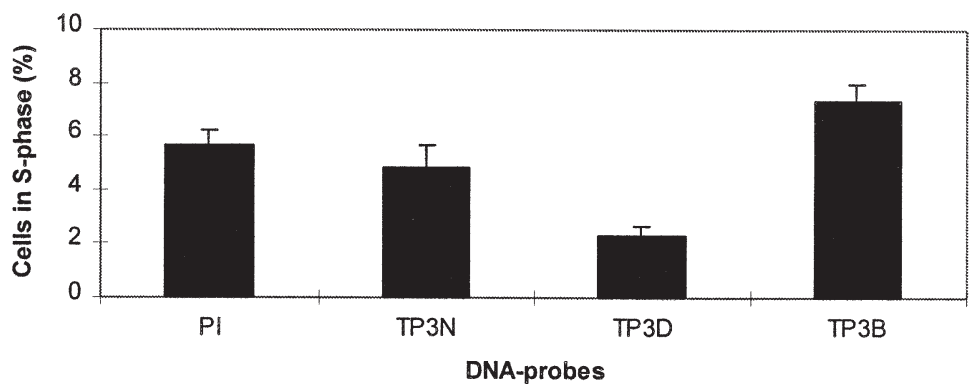

Fig. 1. Cells in S-phase measured with PI or TP3 $48 \mathrm{~h}$ following tape stripping (TP3N: keratin 10-negative population, TP3D: keratin 10-dim population, TP3B: keratin 10-bright population).

Table 1

Correlation coefficients and $\beta$-coefficients for proliferation measured with TP3 in different epidermal subpopulations

\begin{tabular}{lcclrc}
\hline & PI & TP3N & TP3D & TP3B & $\beta$-coefficient \\
\hline PI & 1.00 & $0.85^{*}$ & 0.40 & -0.24 & \\
TP3N & $0.85^{*}$ & 1.00 & $0.44^{*}$ & -0.20 & 0.846 \\
TP3D & 0.40 & $0.44^{*}$ & 1.00 & 0.17 & -0.008 \\
TP3B & -0.24 & -0.20 & 0.17 & 1.00 & \\
\hline
\end{tabular}

TP3N: keratin 10-negative population, TP3D: keratin 10-dim population, TP3B: keratin 10-bright population.

(strongly positive) based on their fluorescence. The percentages of cells in S- and $\mathrm{G}_{2} \mathrm{M}$-phase were determined with Multicycle ${ }^{\mathrm{TM}}$ software (Phoenix Flow Systems, San Diego, CA, USA). This program can be used to derive cell cycle parameters from DNA content histograms. The method is based on recognising that the cell cycle histogram is produced as a result of Gaussian broadening of the theoretically perfect distribution. By fitting the $\mathrm{G}_{1}$ - and $\mathrm{G}_{2}$-peaks as Gaussian curves and the $\mathrm{S}$-phase distribution as a Gaussian-broadened distribution, the underlying distribution of cells over the cell cycle can be recovered. The standard background subtraction, as adjusted in Multicycle ${ }^{\mathrm{TM}}$, was used. DNA-content was measured with TP3 in each of the keratin 10-subpopulations, whereas PI was used to measure the overall DNA content, i.e., the DNA content of all subpopulations together.

\subsection{Statistical analysis}

Data with respect to proliferation were statistically tested with multiple regression analysis, and a correlation coefficient was determined. One- and two-way repeated ANOVA tests were used to determine differences in number of proliferating cells. A two-way repeated ANOVA test was also used to analyse differences in the number of keratin 10 expressing and vimentin-positive cells.

\section{Results}

Proliferation characteristics (percentage of epithelial cells in S-phase) measured in the entire epidermal cell population with PI and those measured in different epidermal subpopulations with TP3 are shown 
(A)
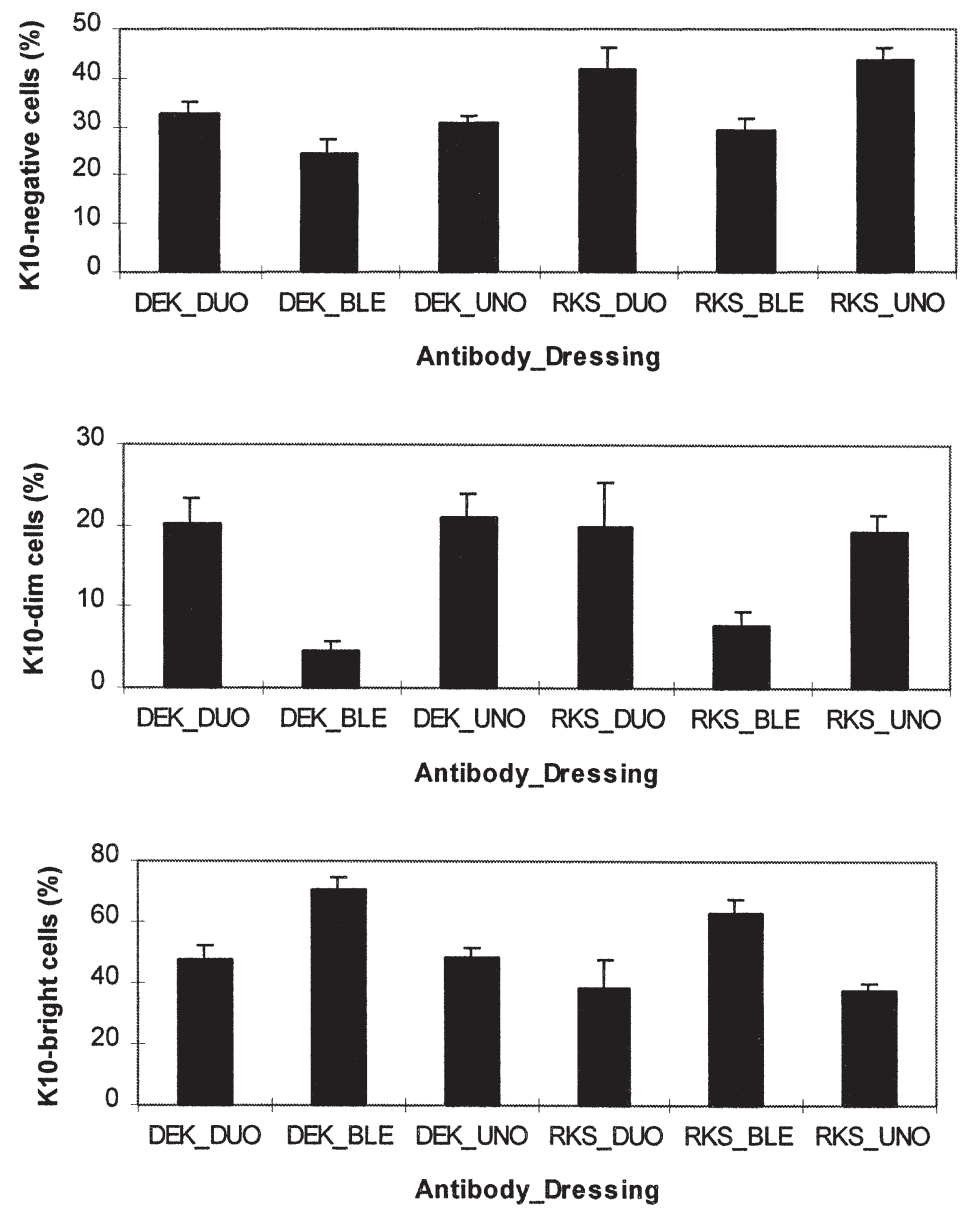

Fig. 2. (A): Percentages of keratin 10-negative cells $48 \mathrm{~h}$ following tape stripping; unoccluded (UNO) or after occlusion with Duoderm (DUO) or Blenderm (BLE) measured with RKSE 60 (RKS) and DE-K10 (DEK). (B): Percentages of keratin 10-dim cells $48 \mathrm{~h}$ following tape stripping; unoccluded (UNO) or after occlusion with Duoderm (DUO) or Blenderm (BLE) measured with RKSE 60 (RKS) and DE-K10 (DEK). (C): Percentages of keratin 10-bright cells $48 \mathrm{~h}$ following tape stripping; unoccluded (UNO) or after occlusion with Duoderm (DUO) or Blenderm (BLE) measured with RKSE 60 (RKS) and DE-K10 (DEK).

in Fig. 1. It can be seen that proliferation in the keratin 10-negative population measured with TP3 approximates proliferation in the whole population measured with PI. In Table 1 it can be seen that the correlation of measured proliferation in the keratin 10-dim and -bright population is below the level of statistical significance. The measured proliferation in the keratin 10-bright population has a reciprocal relation with that measured with PI. A multiple regression analysis showed that the $\beta$-coefficient of the measured proliferation in the keratin 10-dim population was so low that addition of the measured proliferation of this subpopulation to that of the keratin 10-negative compartment did not result in a better approximation of the proliferation measured with PI (Table 1).

Differentiation characteristics were measured with both DE-K10 as well as with RKSE60. In the keratin 10-negative compartment, only the antibody used $(p<0.01)$, and not the kind of occlusion, made a statistically significant difference with respect to the percentage of measured keratin 10-negative cells (Fig. 2A). This percentage was higher when RKSE60 was used. This observation did not hold for the keratin 10-dim subpopulation; in this compartment only the dressing used made a statistically significant 
(A)

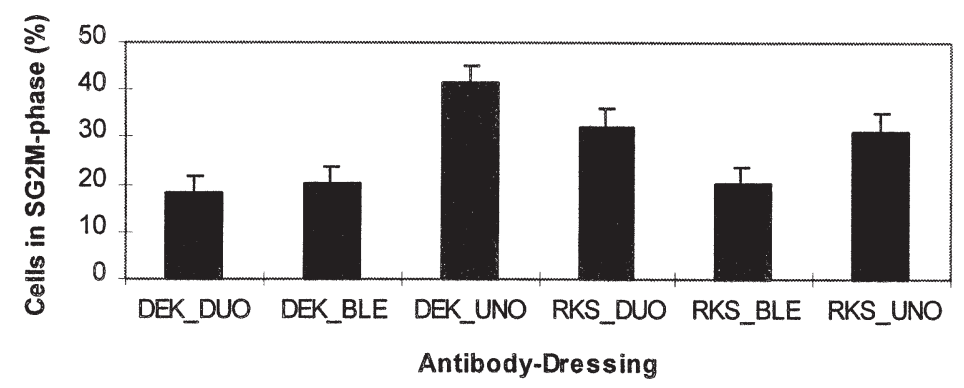

(B)

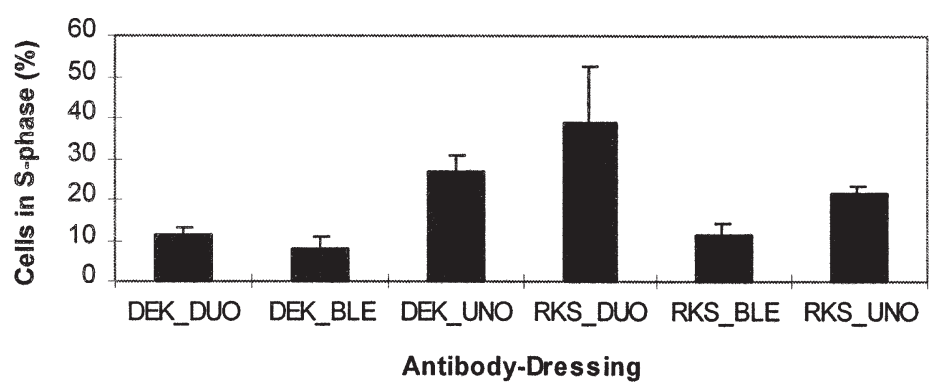

Fig. 3. (A) Percentages of cells in $\mathrm{SG}_{2} \mathrm{M}$-phase in the keratin 10-negative compartment $48 \mathrm{~h}$ following tape stripping; unoccluded (UNO) or after occlusion with Duoderm (DUO) or Blenderm (BLE). (B) Percentages of cells in S-phase in the keratin 10-negative compartment $48 \mathrm{~h}$ following tape stripping; unoccluded (UNO) or after occlusion with Duoderm (DUO) or Blenderm (BLE).

difference $(p<0.01)$ in the number of keratin 10-dim cells (Fig. 2B). Blenderm occlusion resulted in a lower number $(p<0.01)$ of keratin 10-dim cells than did Duoderm (means for Blenderm: 4.5 (DEK10)/7.9 (RKSE60) and Duoderm: 20.1 (DE-K10)/20.0 (RKSE60)). The number of keratin 10-dim cells in skin covered with Blenderm was also lower $(p<0.01)$ than that in uncovered skin (means for Blenderm: 4.5 (DE-K10)/7.9 (RKSE60) and for unoccluded skin: 21.1 (DE-K10)/19.4 (RKSE60)). In the keratin 10-bright cells, both the antibody as well as the occlusion made a statistically significant difference ( $p<0.01$ and $p<0.05$, respectively); when DE-K10 was used the number of bright cells was higher than with the use of RKSE60 (Fig. 2C). Occlusion with Duoderm resulted in a lower percentage $(p<0.05)$ of keratin 10-bright cells than occlusion with Blenderm (means for Duoderm: 47.3 (DEK10)/38.8 (RKSE60) and for Blenderm: 70.8 (DE-K10)/63.4 (RKE60)). In comparing Blenderm to unoccluded skin, the number of keratin 10-bright cells was lower $(p<0.05)$ in unoccluded skin (means for Blenderm: 70.8 (DE-K10)/63.4 (RKSE60) and for unoccluded skin: 48.3 (DE-K10)/37.4 (RKSE60)).

Assessment of proliferation (cells in $\mathrm{SG}_{2} \mathrm{M}$-phase) in the keratin 10-negative compartment showed that neither the antibody nor the kind of dressing made a statistically significant difference in the number of proliferating cells (Fig. 3A). Concerning exclusively the S-phase cells, a difference in the percentage of these cells, caused by different dressings, was found in the cells stained with DE-K10 $(p<0.01)$ but not in those stained with RKSE60 (Fig. 3B). In the DE-K10-group, the unoccluded skin contained more $(p<0.01)$ cells in S-phase than skin occluded with Duoderm or Blenderm (means for Duoderm: 11.4, for Blenderm: 7.9, and for unoccluded skin: 26.7).

Inflammation parameters were not statistically significant different in the occluded and unoccluded groups and were of the same magnitude as in normal skin. 


\section{Discussion}

In the present study, proliferation and differentiation characteristics in epidermal subpopulations of normal skin, uncovered or covered with one of the two kinds of dressing, were assessed $48 \mathrm{~h}$ after tape stripping. The subpopulations were defined by their different expression of keratin 10, and measured with two antibodies; DE-K10 and RKSE60.

PI has been used widely for a long time in order to measure proliferation $[6,15,19]$. In this study we demonstrated that proliferation in the keratin 10-negative, vimentin-negative epidermal compartment as measured with TP3 is a good approximation of proliferation in the total compartment as measured with PI. Proliferation measured in the keratin 10-dim and keratin 10-bright compartment did not make a substantial contribution to this approximation. This can be explained by the fact that the number of keratin 10-dim cells is very low so that they do not have an important contribution to proliferation. The keratin 10-bright cells, differentiated cells that have lost the ability to divide, were not supposed to divide any more, which was seen in the DNA-histograms that were unreliable because of high coefficients of variation (CVs). Probably this is a matter of false positive results with TP3 caused by different binding properties resulting in a high CV for differentiated cells, which was not seen with the use of PI. Although the keratin 10-dim population might play a role in some phase of epidermal regeneration, the present study suggests that the keratin 10-dim population is irrelevant in stimulated hyperproliferation after tape stripping. Proliferation in the keratin 10-bright compartment also proved to be not relevant in this context. This means that it is not necessary to measure proliferation after tape stripping in all cells: only proliferation in the keratin 10-negative compartment is relevant.

The percentage of keratin 10-negative cells in S-phase only differed between unoccluded, Blenderm and Duoderm occluded skin measured with DE-K10 and not in those measured with RKSE60. This phenomenon can be explained by the fact that the keratin 10-negative population as measured with DE$\mathrm{K} 10$ is smaller and so more accurate, i.e., only comprises negative and no dim cells. This implicates that the percentage of S-phase cells is higher, so that in this population the kind of occlusion makes a difference: the more occlusive the dressing, the less cells in S-phase were present.

In contrast to the cells in S-phase, no differences between occluded and unoccluded stripped skin were found in the $\mathrm{SG}_{2} \mathrm{M}$-compartment. This can be explained by the synchronising effect of tape stripping on recruited cells; all cells are in S-phase and none of them has already reached $\mathrm{G}_{2}$-phase $48 \mathrm{~h}$ after tape stripping. From previous studies it is known that hyperproliferation is induced in normal skin 40 to $48 \mathrm{~h}$ after tape stripping $[7,12,22,23]$. The statistically significant effect that was seen in the S-compartment could not be shown in the $\mathrm{SG}_{2} \mathrm{M}$-compartment, because addition of $\mathrm{G}_{2}$-cells did not make a relevant contribution and only diluted the effect of the difference in the S-compartment.

The number of cells in the different subpopulations (less keratin 10-negative cells, more bright cells, and the same number of dim cells) measured with DE-K10 can be an indication that this antibody detects positive cells in a more sensitive way than RKSE60 does. The number of negative cells is lower, because the most fluorescent cells of this population are classified as dim cells. As the most fluorescent dim cells were classified as bright cells, the percentage of dim cells remains the same as with RKSE60. Another possible explanation is that DE-K10 causes more aspecific staining, as a result of which more cells would be false positive. Although it is not clear which of these explanations is the right one, it can be assumed that DE-K10 has no additional value in the determination of the keratin 10-dim population. With respect to occlusion, the differences in the keratin 10-dim and -bright compartment are between unoccluded or partially occluded (Duoderm) skin and maximal occluded (Blenderm) skin. The lower number of keratin 10-dim cells and the higher number of bright cells may be caused by the inhibitory effect of Blenderm 
on cell turnover; cells remain longer in the epidermis, so they have more time to synthesise keratin 10 and the amount of keratin 10 per cell increases. This effect was not seen with the less occlusive Duoderm and in unoccluded skin.

Inflammation characteristics in this study were not different from unoccluded and occluded skin. This was also found in earlier studies [14,24].

Data with respect to proliferation and differentiation characteristics show that proliferation parameters are more responsive to occlusion than differentiation parameters. Unoccluded skin and Blenderm (maximal occlusive) are the extremes in the spectrum of occlusion, whereas the partially occlusive dressing Duoderm is intermediate between these two. Differences in number of proliferating cells were already present in samples covered with Duoderm (intermediate occlusion), whereas differences in percentage of differentiated cells were only present in samples covered with Blenderm (maximal occlusion).

It is known that psoriatic skin contains relatively less differentiated cells [12] and more proliferative cells than normal skin, although the total number of both cell populations per unit of surface is increased. The beneficial effect of occlusive treatment on various skin diseases, such as psoriasis is well-known; occlusion stimulates regeneration of the disturbed granular layer [11], and reduces the mitotic rate [4, 11,24]. The inhibitory effect of occlusion on proliferation was shown before in animal skin. A previous study in mice reported that occlusion inhibited the proliferative response after tape stripping [18]. Just as in the present study, water vapour-impermeable foil prevented an increase in DNA synthesis more than did partially occlusive wraps, which were more effective than the vapour-permeable foil. The results with regard to the effect of occlusion on tape stripped human skin are not uniform. Van de Kerkhof et al. found no differences with respect to number of cells in $\mathrm{SG}_{2} \mathrm{M}$-phase between uncovered and occluded skin $48 \mathrm{~h}$ after tape stripping [22]. This is in contrast with the findings of Fischer et al.; they showed that occlusive dressings decreased the mitotic activity of human skin $72 \mathrm{~h}$ after tape stripping [8]. The inhibitory effect of occlusion on mitotic activity in stripped skin was also found in another study [25]. In contrast to the present study, Fischer et al. showed that the material need not to be highly occlusive, as the adhesive backing was equally effective as occlusive dressing, porous (semi-occlusive) tape and plastic film in inhibiting mitotic rate $[9,10]$.

From the results of this study, it can be concluded that multi parameter flow cytometry is an adequate approach to evaluate epidermal proliferation and differentiation in vivo. Occlusion causes a decrease of stimulated hyperproliferation compared to unoccluded skin. In this respect Blenderm is more effective than Duoderm. This beneficial effect was also seen in markers of differentiation: the number of keratin 10-bright (differentiated) cells was the highest in skin samples that were covered with Blenderm. Therefore, it can be concluded that occlusion, especially maximal occlusion, causes normalisation of hyperproliferative skin.

\section{References}

[1] Z. Bata-Csorgo, C. Hammerberg, J.J. Voorhees and K.D. Cooper, Flow cytometric identification of proliferative subpopulations within normal human epidermis and the localization of the primary hyperproliferative population in psoriasis, J. Exp. Med. 178 (1993), 1271-1281.

[2] F.W. Bauer and J.B. Boezeman, Flow cytometric methods in human skin with respect to cell cycle kinetics, in: Psoriasis: Cell Proliferation, N.A. Wright and R.S. Camplejohn, eds, Churchill Livingstone, Edinburgh, 1983, pp. 104-116.

[3] F.W. Bauer, N.H. Crombag, R.M. de Grood and G.J. de Jongh, Flow cytometry as a tool for the study of cell kinetics in epidermis, Br. J. Dermatol. 102 (1980), 629-639.

[4] D.L. Baxter and R.B. Stoughton, Mitotic index of psoriatic lesions treated with anthralin, glucocorticosteriod and occlusion only, J. Invest. Dermatol. 54 (1970), 410-412. 
[5] J.B. Boezeman, F.W. Bauer and R.M. de Grood, Flow cytometric analysis of the recruitment of G0 cells in human epidermis in vivo following tape stripping, Cell Tissue Kinet. 20 (1987), 99-107.

[6] H.A. Crissman and J.A. Steinkamp, Rapid simultaneously measurement of DNA, protein and cell volume in single cells from large mammalian cell populations, J. Cell Biol. 59 (1973), 766-771.

[7] S. de Mare, P.E. van Erp, F.C. Ramaekers and P.C. van de Kerkhof, Flow cytometric quantification of human epidermal cells expressing keratin 16 in vivo after standardized trauma, Arch. Dermatol. Res. 282 (1990), 126-130.

[8] L.B. Fisher and H.I. Maibach, The effect of occlusive and semipermeable dressings on the mitotic activity of normal and wounded human epidermis, Br. J. Dermatol. 86 (1972), 593-600.

[9] L.B. Fisher and H.I. Maibach, Physical occlusion controlling epidermal mitosis, J. Invest. Dermatol. 59 (1972), 106-108.

[10] L.B. Fisher, H.I. Maibach and R.J. Trancik, Variably occlusive tape systems and the mitotic activity of stripped human epidermis. Effects with and without hydrocortisone, Arch. Dermatol. 114 (1978), 727-729.

[11] L. Fry, J. Almeyda and R.M. McMinn, Effect of plastic occlusive dressings on psoriatic epidermis, Br. J. Dermatol. 82 (1970), 458-462.

[12] C.P. Glade, B.A. Seegers, E.F. Meulen, C.A. van Hooijdonk, P.E. van Erp and P.C. van de Kerkhof, Multiparameter flow cytometric characterization of epidermal cell suspensions prepared from normal and hyperproliferative human skin using an optimized thermolysin-trypsin protocol, Arch. Dermatol. Res. 288 (1996), 203-210.

[13] C.P. Glade, P.E. van Erp, C.A. van Hooijdonk, M.E. Elbers and P.C. van de Kerkhof, Topical treatment of psoriatic plaques with 1 alpha, 24 dihydroxyvitamin D3: a multiparameter flow cytometrical analysis of epidermal growth, differentiation and inflammation, Acta Derm. Venereol. 75 (1995), 381-385.

[14] A.B. Gottlieb, L. Staiano-Coico, S.R. Cohen, M. Varghese and D.M. Carter, Occlusive hydrocolloid dressings decrease keratinocyte population growth fraction and clinical scale and skin thickness in active psoriatic plaques, J. Dermatol. Sci. 1 (1990), 93-96.

[15] A. Krishan, Rapid flow cytofluorometric analysis of mammalian cell cycle by propidium iodide staining, J. Cell Biol. 66 (1975), 188-193.

[16] M. Laporte and M. Heenen, The heterogeneity of the germinative compartment in human epidermis and its implications in pathogenesis, Dermatology 189 (1994), 340-343.

[17] H. Pinkus, Tape stripping in dermatological research. A review with emphasis on epidermal biology, G. Ital. Dermatol. Minerva. Dermatol. 107 (1966), 1115-1126.

[18] E. Proksch, W.M. Holleran, G.K. Menon, P.M. Elias and K.R. Feingold, Barrier function regulates epidermal lipid and DNA synthesis, Br. J. Dermatol. 128 (1993), 473-482.

[19] P.S. Rabinovitch, R.M. Torres and D. Engel, Simultaneous cell cycle analysis and two-color surface immunofluorescence using 7-amino-actinomycin D and single laser excitation: applications to study of cell activation and the cell cycle of murine Ly-1 B cells, J. Immunol. 136 (1986), 2769-2777.

[20] M.B. Randall, K.R. Geisinger, T.E. Kute, D.H. Buss and R.W. Prichard, DNA content and proliferative index in cutaneous squamous cell carcinoma and keratoacanthoma, Am. J. Clin. Pathol. 93 (1990), 259-262.

[21] L. Staiano-Coico, Z. Darzynkiewicz and C.K. McMahon, Cultured human keratinocytes: discrimination of different cell cycle compartments based upon measurement of nuclear RNA or total cellular RNA content, Cell Tissue Kinet. 22 (1989), 235-243.

[22] P.C. van de Kerkhof, S. de Mare, W.P. Arnold and P.E. van Erp, Epidermal regeneration and occlusion, Acta Derm. Venereol. 75 (1995), 6-8.

[23] P.C. van de Kerkhof, H. van Rennes, R.M. de Grood, G.J. de Jongh, F.W. Bauer and P.D. Mier, Response of the clinically uninvolved skin of psoriatic patients to standardized injury, Br. J. Dermatol. 109 (1983), 287-294.

[24] I.M. van Vlijmen-Willems, A. Chang, J.B. Boezeman and P.C. van de Kerkhof, The immunohistochemical effect of a hydrocolloid occlusive dressing (DuoDERM E) in psoriasis vulgaris, Dermatology 187 (1993), 257-262.

[25] M.G. Williams and R. Hunter, Studies on epidermal regeneration by means of the strip method, J. Invest. Dermatol. (1957), 407-413 


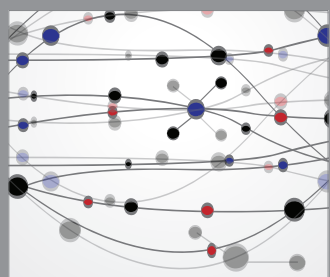

The Scientific World Journal
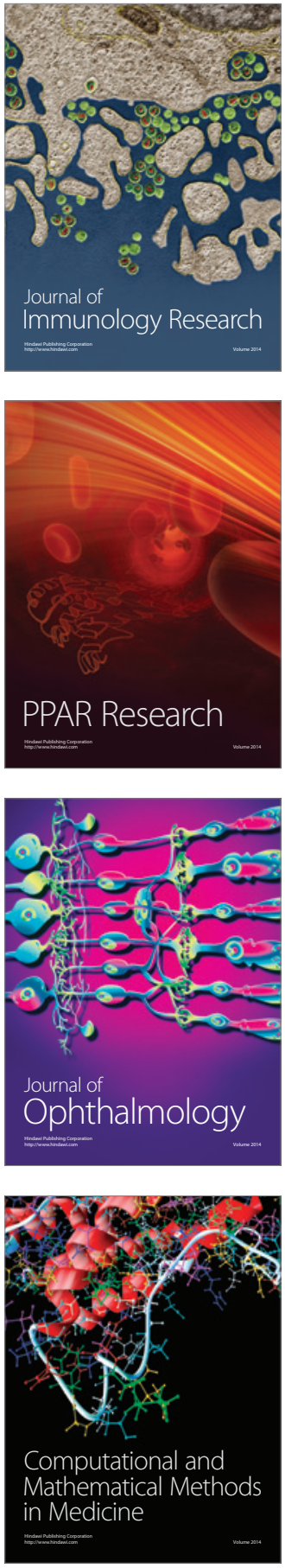

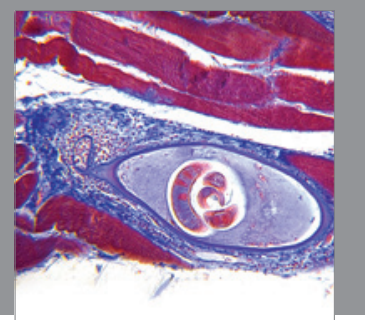

Gastroenterology

Research and Practice
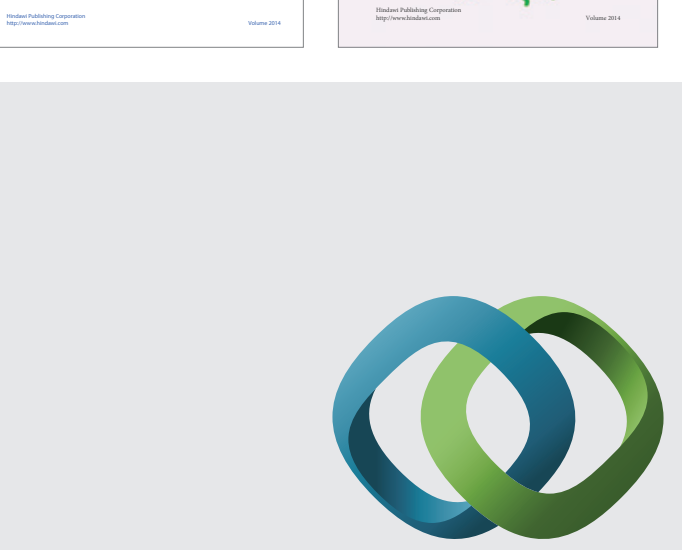

\section{Hindawi}

Submit your manuscripts at

http://www.hindawi.com
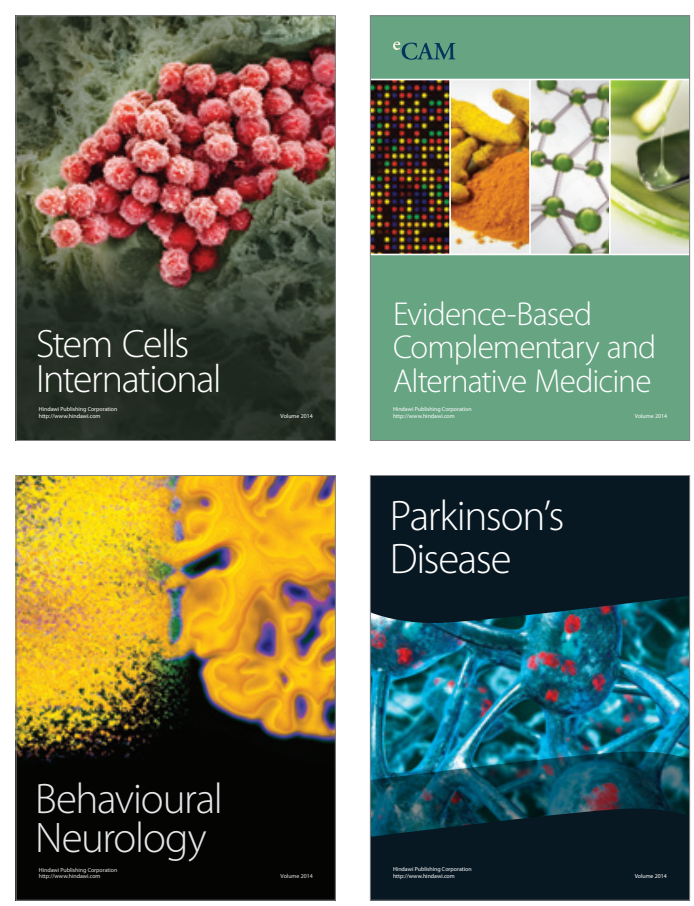

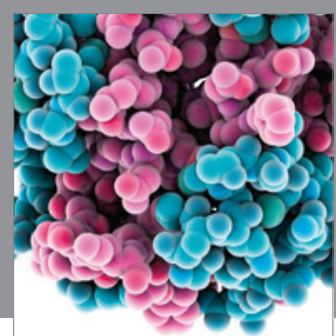

Journal of
Diabetes Research

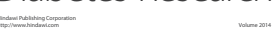

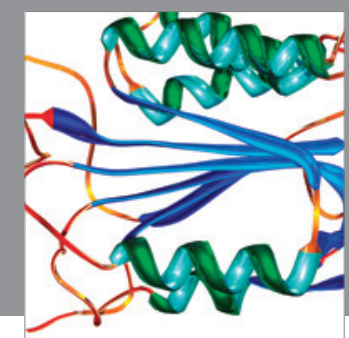

Disease Markers
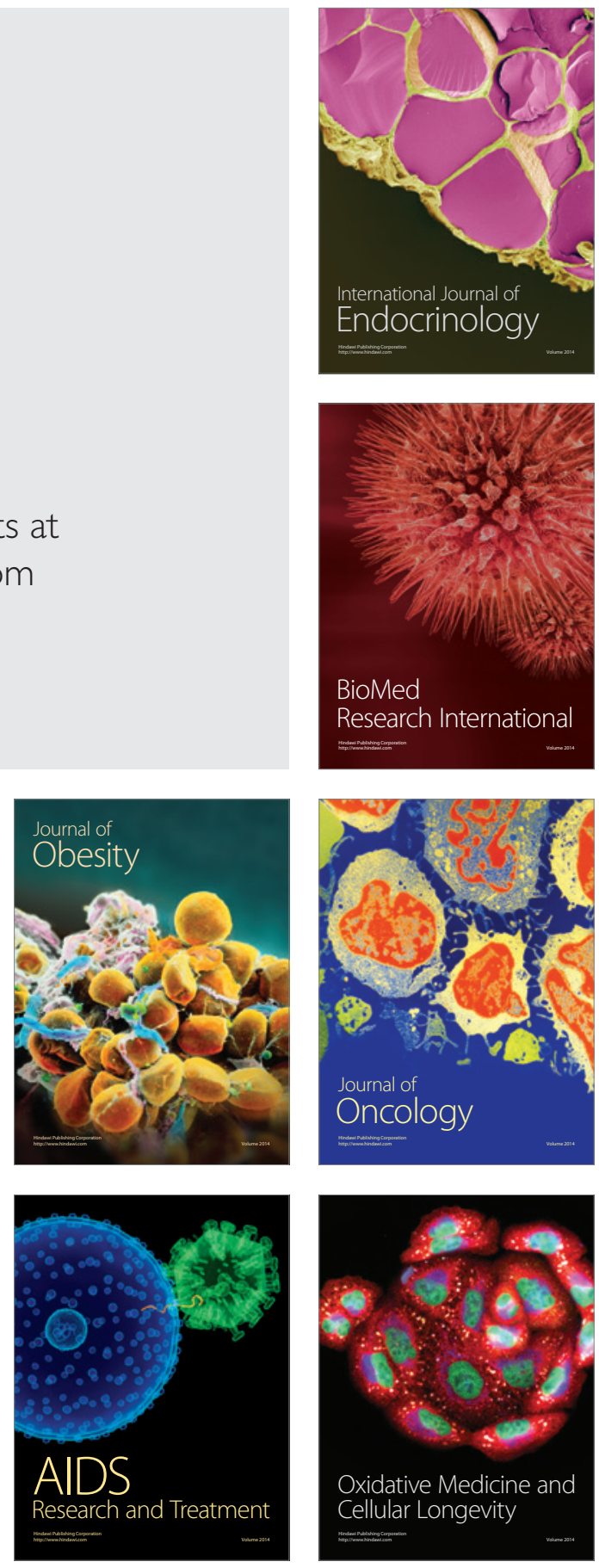